\title{
Aprendizaje en las matemáticas. La gamificación como nueva herramienta pedagógica
}

Learning in mathematics. Gamification as a new pedagogical tool.

aprendizagem em matemática. Gamificação como uma nova ferramenta pedagógica.

\section{ARTÍCULO GENERAL}

\section{Marleni Mamani Sanchez mmamanis@ucvvirtual.edu.pe}

https://0000-0002-1458-6463

Recibido 25 de Diciembre 2021 | Arbitrado y aceptado 25 de Diciembre 2021 | Publicado el 28 de Enero 2022

\section{RESUMEN}

El objetivo general de esta investigación se basa en considerar la gamificación como nueva herramienta pedagógica para el aprendizaje de las matemáticas. La metodología empleada se basó en un diseño bibliográfico con un tipo de investigación documental. Como conclusión se puede indicar que la gamificación resulta interesante e importante para mejorar el aprendizaje en todos los niveles educativos pues ayuda en gran medida a la motivación de los estudiantes, así como la disminución del estrés presente en ellos producto del miedo a afrontar las clases que les parecen aburridas o difíciles $y$ de diversos factores como socioculturales y económicos. A pesar de que la gamificación posee desventajas en cuanto a la poca accesibilidad de la tecnología tanto para el estudiante como para los docentes y la poca habilidad tecnológica que tienen algunos, sus ventajas son mayores y generan aspectos positivos en el incremento del aprendizaje de los estudiantes. En cuanto a la implementación de la gamificación en las matemáticas se ha observado una buena influencia de esta herramienta para desarrollar habilidades de cálculos y lógica matemática en los estudiantes pues les permite a través del uso de los diferentes software existentes desarrollar estrategias que les permitan avanzar de nivel, lograr cumplir las metas, con el propósito de alcanzar las mejores calificaciones los que los motiva a seguir siempre buscando mejorar sus capacidades para comprender cada tema de la materia.

Palabras clave: gamificación, tecnología, herramienta, estudiante, docente.

\section{ABSTRACT}

The general objective of this research is based on considering gamification as a new pedagogical tool for learning mathematics. The methodology used was based on a bibliographic design with a type of documentary research. In conclusion, it can be indicated that gamification is interesting and important to improve learning at all educational levels, since it greatly helps to motivate students, as well as the reduction of stress present in them as a result of the fear of facing the classes that They seem boring or difficult and of various factors such as sociocultural and economic. Despite the fact that gamification has disadvantages in terms of the low accessibility of technology for both students and teachers and the little technological ability that some have, its advantages are greater and generate positive aspects in increasing student learning. . Regarding the implementation of gamification in mathematics, a good influence of this tool has been observed to develop calculation skills and mathematical logic in students, since it allows them, through the use of the different existing software, to develop strategies that allow them to advance from level, achieve the goals, with the purpose of achieving the best grades, which motivates them to always continue seeking to improve their abilities to understand each topic of the subject.

Keywords: gamification, technology, tool, student, teacher.

\section{RESUMO}

O objetivo geral desta pesquisa baseia-se em considerar a gamificação como uma nova ferramenta pedagógica para o aprendizado da matemática. A metodologia utilizada foi baseada em um desenho bibliográfico com um tipo de pesquisa documental. Em conclusão, pode-se indicar que a gamificação é interessante e importante para melhorar o aprendizado em todos os níveis de ensino, pois auxilia muito na motivação dos alunos, bem como na redução do estresse presente neles em decorrência do medo de enfrentar as aulas que Parecem chatos ou difíceis e de vários fatores como socioculturais e econômicos. Apesar da gamificação apresentar desvantagens em termos de baixa acessibilidade da tecnologia tanto para alunos quanto para professores e a pouca habilidade tecnológica que alguns possuem, suas vantagens são maiores e geram aspectos positivos no aumento da aprendizagem dos alunos. Em relação à implementação da gamificação na matemática, observou-se uma boa influência desta ferramenta para desenvolver habilidades de cálculo e lógica matemática nos alunos, pois permite que eles, através do uso dos diferentes softwares existentes, desenvolvam estratégias que lhes permitam avançar de nível, atingir as metas, com o propósito de obter as melhores notas, o que os motiva a continuar sempre buscando aprimorar suas habilidades de compreensão de cada tópico da disciplina. Palavras-chave: gamificação, tecnologia, ferramenta, aluno, professor. 


\section{Introducción}

Todas las especies tienen capacidades de aprendizaje para adaptarse al ambiente que les rodea, y poder desenvolverse y perdurar en el tiempo. En el caso del ser humano desde su nacimiento está en constante aprendizaje desarrollando sus capacidades cognitivas, aprendiendo aspectos que le servirán a lo largo de su vida; al llegar a su etapa escolar adquiere habilidades para entender la información impartida de manera que pueda procesar los conocimientos necesarios y poder avanzar en su periodo educacional. Tal como lo menciona (Aguado-Aguilar, 2001):

La adaptación de la conducta al ambiente esta medida por procesos perceptivos, cognitivos y de organización motora. De forma simplificada, ello significa que el sistema biológico al que llamamos mente o cerebro debe "procesar" los estímulos del ambiente, comparar el resultado de ese procesamiento con el conocimiento anterior y organizar un "output", es decir, una "salida", o respuesta motora a estos estímulos (pág. 373)

El aprendizaje es un proceso que viene dado no solo por la capacidad cognitiva que posea el estudiante, se refiere también a la disposición y motivación que tenga la persona de aprender y los procesos de planificación y control que se tenga para impartir el conocimiento, (Valle, González, Cuevas, \& Fernández, 1998, pág. 54), por lo que todos estos aspectos son importantes para considerar que las estrategias de aprendizaje son adecuadas para poder garantizar que el proceso de aprendizaje sea desarrollado correctamente logrando el objetivo.

En ocasiones se observa que la poca capacidad de resolver problemas cotidianos por parte de los estudiantes se debe a un aprendizaje significativo y desarrollo cognitivo conceptual deficiente, (Martínez, Arrieta, \& Meleán, 2012), que debe tratarse para mejorar estos aspectos y lograr con éxito que el estudiante aprenda de manera adecuada, y que los conduzca a tener éxito en sus estudios.

Una de las teorías del aprendizaje que existe es la propuesta por Ausubel en el año 2002 denominada aprendizaje significativo, "esta es un proceso a través del cual una información se relaciona, de manera no arbitraria ni literal, con un aspecto relevante de la estructura cognitiva del individuo" (Martínez, et al., 2012), esta es una teoría psicológica cuyo fin es aprender en el aula y a su vez para que se produzca un aprendizaje significativo debe tomarse en cuenta dos criterios importantes: 
- Disposición (motivación y actitud) de aprendizaje significativo por parte del alumno.

- Presentación de un material potencialmente significativo: a su vez esta última condición supone que:

○ El material de aprendizaje posea un significado lógico y se pueda relacionar con cualquier estructura cognitiva apropiada y pertinente.

- La estructura cognitiva del sujeto concreto contenga ideas de anclaje adecuadas, con las que el nuevo material pueda interactuar. (pág. 37)

De igual manera existe otra teoría denominada Teoría de los campos conceptuales de Gerard Vergnaud propuesta en el año 1980, esta surge de los legados de Piaget donde ideas de adaptación, desequilibración, re-equilibración y la gran piedra angular que es el concepto de esquema. (Martínez,et al., 2012). De igual manera se le debe reconocer los aportes mostrados por Vygostky donde muestra la importancia de la interacción social, al lenguaje, y a la simbolización en el dominio progresivo de un campo conceptual.

Para Vergnaud un concepto está conformado por tres aspectos diferentes que es la referencia, el significado y el significante, para lograr la comprensión de un concepto se debe estructurar un esquema que permita al estudiante entender lo impartido en el aula. (Martínez, et al., 2012) En la Tabla 1 se demuestra cómo es la composición de los esquemas que puede ser único para cada estudiante y se puede modificar en caso de que sea ineficaz.

\section{Tabla 1}

Composición de los esquemas

\section{ASPECTOS}

ANTICIPACIONES:

Ayudan a lograr fines, efectos y etapas intermedias a la planificación y control de la acción

REGLAS DE ACCION:

Son del tipo si...entonces... permiten generar la serie de acciones del aprendiz.

INVARIANTES OPERATORIOS:

Dirigen el reconocimiento de los elementos cognitivos de la situación y la recolección de la información sobre el problema a resolver. Pueden ser: Conceptos-en-acción Teoremas-en-acción

\section{INFERENCIAS O RAZONAMIENTOS:}

Permiten deducir las reglas de acción y las anticipaciones a partir de las informaciones y del sistema de invariantes operatorios que posee el aprendiz.

Fuente: (Martínez, et al.,2012) 
La memoria y el aprendizaje están relacionados ya que lo aprendido queda grabado en el cerebro y está en constante reorganización dependiendo de la cantidad de conocimiento que el sujeto va adquiriendo (Aguado-Aguilar, 2001).

Para que el aprendizaje sea adecuado se deben aplicar herramientas pedagógicas que permitan una buena comprensión, estas vienen estrechamente relacionadas con ciertas características que debe tener el docente para lograr mejorar la educación en cualquier nivel, por lo que la comunicación docente-alumno debe ser eficaz (del Barrio \& García, 2006), es así como en todo proceso de comunicación es importante tomar en cuenta que la voz, la personalidad y el lenguaje que utiliza quien comunica las use correctamente. En la Tabla 2 se detallan estos tres aspectos.

Dentro de las muchas herramientas pedagógicas aplicadas en las aulas de clases a nivel infantil y de primaria se puede nombrar los cuentos motores que permiten al estudiante a través de la narración, la música, los juegos, las simulaciones, y dramatizaciones a desarrollar sus capacidades motoras (Iglesia, 2008), de igual manera permite que los niños desarrollen su capacidad creativa, e interdisciplinar diversas áreas como la música y las artes plásticas.

Adicionalmente se menciona el juego lingüístico, esta herramienta pedagógica es utilizada en el aula para la comprensión de lenguas extranjeras, y como menciona (Díaz, 2012) permiten pues, que el alumno y el profesor tengan una relación diferente a la que crean en un aula, y así el docente está en contacto más directo con los alumnos permitiendo ser más amena la clase facilitando el proceso enseñanza-aprendizaje.

\section{Tabla 2}

Características del proceso de la comunicación

\begin{tabular}{cc}
\hline ASPECTOS A & CARCATERISTICAS \\
CONSIDERAR & -Claridad. Las palabras tienen que ser claras. Hay que mover más la lengua \\
& para hablar claro. \\
& -Volumen adecuado al contexto. Se debe conseguir mucha resonancia para \\
& que las palabras tengan más consistencia. \\
& -Ritmo adecuado. La velocidad del habla normal es de 150 palabras por \\
En cuanto a la & minuto. Una velocidad lenta aporta monotonía, una excesivamente rápida \\
voz y las & provoca cansancio, confusión y aturdimiento. \\
palabras: & -Ayudarse del cuerpo. La expresión corporal, el gesto, crea las pausas cuando \\
& hablamos y transmite intenciones. Hablar es una acción de todo el cuerpo. \\
& La mayoría de las veces, incluso, dice más el cuerpo que las propias \\
& palabras. \\
& -Saber jugar con la voz. Hay que hablar manifestando nuestras intenciones, \\
& subiendo y bajando los tonos, jugando con la duración de las vocales y \\
& con las pausas, es decir, dar vida, dotar de intenciones al mensaje. \\
\hline
\end{tabular}


Marleni Mamani Sanchez

\begin{tabular}{cc}
\hline & -Autoestima. Para comunicar es fundamental tener una valoración de uno \\
mismo. & -Empatía. Es necesario atender y hacer ver que entendemos la situación del \\
otro, sin tener que identificarnos necesariamente con ella, pero intentando & "ponernos en su lugar" \\
En cuanto a la & -Sintonía. Tan importante como el mensaje es el interés que tiene en ello el \\
personalidad & que lo escucha. \\
& -Autenticidad. Sólo cuando creemos en lo que estamos comunicando \\
podemos transmitir convicción e influir en los demás. & \\
& -Autocontrol. Una comunicación eficaz y positiva implica, también, un \\
adecuado nivel de ejecución. \\
-Asertividad, entendida como la propia capacidad de autoafirmación, de \\
expresión directa de los propios sentimientos, opiniones, derechos, etc. \\
\hline -Preparación. La clave del éxito se asienta en la preparación pausada. \\
-Corrección. Utilizar un lenguaje adecuado al público, claro y sencillo, pero \\
siempre correcto. \\
-Orden. Mantener el esquema clásico de "Inicio, Desarrollo, Conclusión": es \\
lo que los demás esperan y, además, lo más eficaz. \\
-Claridad de las ideas. Marcar adecuadamente los tiempos. Cada momento del \\
discurso tiene su propia dinámica y es preciso respetarla. \\
-Novedad. Ser creativo, original, sobre todo al principio, para ganar la \\
atención, para motivar y cautivar.
\end{tabular}

Fuente: (del Barrio \& García, 2006)

De igual manera los juegos para aprender matemáticas son una de las herramientas utilizadas para mejorar la comprensión numérica, debido a que esta asignatura es considerada compleja y aburrida por muchos estudiantes (Muñiz, Alonso, \& Rodríguez, 2014), de manera que se deben aplicar diversas herramientas pedagógicas que permitan el entendimiento y se les brinda la capacidad de resolver problemas matemáticos a los estudiantes con técnicas de aprendizaje adaptadas a las necesidades de cada estudiante.

Producto de los cambios constantes en el mundo y en la educación las estrategias de aprendizaje y herramientas pedagógicas han evolucionado y en conjunto con las TIC han permitido llegar de diversas maneras a cada alumno para lograr que adquieran los conocimientos necesarios para su desarrollo en todos los niveles educativos.

Estos avances tecnológicos no son nuevos, sino que se han venido abriendo camino a lo largo de los años en la búsqueda de nivelarse con las exigencias conceptuales y pedagógicas de cada época (Facundo, 2004), sin embargo es importante resaltar que no en todas partes del mundo esto es cierto puesto que los países desarrollados están en ventaja con los países en vía de desarrollo, debido a la accesibilidad tecnológica que los primeros tienen con respecto a los segundos.

Es importante destacar que siempre se debe estar en constante evolución y adaptación con estas nuevas tecnologías para aprovechar el interés que tienen los alumnos por ellas y 
adaptarlas al proceso enseñanza-aprendizaje, que permita mejorar cada día en el aspecto pedagógico y en la gerencia educativa, en especial a nivel universitario con el propósito de lograr excelentes profesionales, tal como lo menciona (Briceño, 2014):

La gestión de la educación superior debe considerar entonces, un nuevo punto de vista para la gerencia universitaria, donde se busque operar la gerencia de los saberes y se abandone la noción de las disciplinas como unidades independientes y autónomas, para considerar la noción de sistemas, de multicampus, de la universidad sobre la base de redes de conocimientos propagadas desde las herramientas tecnológicas. (pág. 71)

Para lograr una preparación de calidad del estudiantado es importante también que los docentes sean formados y capacitados de manera que utilicen estas herramientas de forma adecuada en su proceso de docencia y a su vez vayan evolucionando conforme lo haga la tecnología pues la docencia en conjunto con la tecnología es dinámica (Briceño, 2014, pág. 72), si este proceso de capacitación no sucede por falta de interés de las instituciones o de los profesores no se logrará aprovechar las capacidades y habilidades de cada docente para impartir sus conocimientos correctamente.

Las nuevas tecnologías de información y comunicación han sido foco de discusión pues se conocen algunos puntos débiles tanto a nivel de los estudiantes como por parte del profesorado y las instituciones y según (Rodríguez, 2011) son aspectos que se deben tener presentes para la búsqueda constante de posibles soluciones. En la Tabla 3 se expresan estos puntos débiles

\section{Tabla 3}

Problemas con las TIC en la enseñanza educativa

\begin{tabular}{|c|c|}
\hline PROBLEMAS & CARCATERISTICAS \\
\hline \multicolumn{2}{|l|}{ De los estudiantes: } \\
\hline $\begin{array}{l}\text { Acceso restringido a la } \\
\text { tecnología }\end{array}$ & $\begin{array}{l}\text { Es el principal obstáculo existente, ya que sin internet o con mala } \\
\text { conexión no pueden acceder a la tecnología }\end{array}$ \\
\hline $\begin{array}{l}\text { Acceso restringido a } \\
\text { los servicios de apoyo }\end{array}$ & $\begin{array}{l}\text { Poco apoyo técnico genera frustración, atraso y desmotivación al } \\
\text { estudiante }\end{array}$ \\
\hline $\begin{array}{l}\text { Falta de habilidades } \\
\text { tecnológicas }\end{array}$ & $\begin{array}{l}\text { A pesar de ser considerados miembros de la era digital, al } \\
\text { estudiante en ocasiones le cuesta utilizar la tecnología a nivel } \\
\text { educativo, pues carecen de habilidades para manejar wikis, blogs, } \\
\text { podcast, archivos de audio y de video. }\end{array}$ \\
\hline $\begin{array}{l}\text { La falta de la } \\
\text { comunidad }\end{array}$ & $\begin{array}{l}\text { Producto de la misma falta de habilidades tecnológicas aunado a la } \\
\text { timidez de algunos estudiantes, se ven excluidos del apoyo del } \\
\text { resto de los compañeros del grupo }\end{array}$ \\
\hline $\begin{array}{l}\text { Pobres } \\
\text { lectoras }\end{array}$ & $\begin{array}{l}\text { El estudiante con pocas habilidades de lectura les resulta difícil } \\
\text { realizar una redacción adecuada al momento de entregar } \\
\text { asignaciones solicitadas por los docentes pues no tienen la } \\
\text { capacidad de interpretar la información obtenida de internet. }\end{array}$ \\
\hline
\end{tabular}


Marleni Mamani Sanchez

\begin{tabular}{ll}
\hline $\begin{array}{l}\text { Pobres habilidades } \\
\text { académicas }\end{array}$ & $\begin{array}{l}\text { Los estudiantes con pocos hábitos de estudio, poca comprensión en } \\
\text { los tiempos de entrega de las asignaciones, o aquellos que aprenden } \\
\text { de forma visual o auditiva generan atrasos en sus clases. }\end{array}$ \\
\hline $\begin{array}{l}\text { Resistencias de los } \\
\text { estudiantes }\end{array}$ & $\begin{array}{l}\text { Generalmente provocada por la tecnofobia de los estudiantes, falta } \\
\text { de compromiso y falta de competencia. }\end{array}$ \\
\hline Por parte de las instituciones y el profesorado \\
\hline $\begin{array}{l}\text { El uso y no como se usa } \\
\text { Se le da importancia a la disponibilidad tecnológica mas no al } \\
\text { verdadero uso que se le puede dar a ella para el aprendizaje. }\end{array}$ \\
$\begin{array}{l}\text { Falta de habilidades } \\
\text { tecnológicas }\end{array}$ & $\begin{array}{l}\text { Es importante recalcar en el estudiante que es necesario aprender a } \\
\text { diferenciar temas importantes que se obtengan por medio del } \\
\text { internet ya que no solo depende tener habilidades para usar la } \\
\text { tecnología sino saber interpretar la información que esta brinda. }\end{array}$ \\
\hline $\begin{array}{l}\text { Falta de formación del } \\
\text { profesorado } \\
\text { velocidad } \\
\text { cambios }\end{array}$ le los & $\begin{array}{l}\text { Muchas instituciones se resisten a la evolución tecnológica por } \\
\text { causas externas como falta de equipos, de personal que brinde } \\
\text { adiestramientos, entre otros, así como de causas internas como la } \\
\text { falta de disposición del personal para evolucionar con la tecnología } \\
\text { pues consideran que no es la mejor manera de educar, pues aun } \\
\text { tienen el pensamiento tradicionalista y son renuentes al cambio. }\end{array}$ \\
\hline
\end{tabular}

Fuente: (Rodríguez, 2011)

A pesar de las problemáticas antes mencionadas las instituciones están prestas a mejorar la tecnología para que los estudiantes puedan disponer de las herramientas necesarias en estos tiempos cambiantes, y es que las TIC son cada vez mas amigables, accesibles, y adaptables (Castro, Guzmán, \& Casado, 2007, pág. 215), y las instituciones asumen incorporar los equipos tecnológicos con el propósito de poder hacer cambios significativos en la pedagogía y desligarse un poco de la enseñanza tradicional y lograr llegar al aprendizaje más constructivo.

Dentro de las características de las TIC (Castro, et al., 2007) menciona una serie de aspectos importantes como son:

- Inmaterialidad: su materia prima es la información en cuanto a su generación y procesamiento, así se permite el acceso de grandes masas de datos en cortos períodos de tiempo, presentándola por diferentes tipos de códigos lingüísticos y su transmisión a lugares lejanos.

- Interactividad: permite una relación sujeto-maquina adaptada a las características de los usuarios.

- Instantaneidad: facilita que se rompan las barreras temporales y espaciales de las naciones y las culturas.

- Innovación: persigue la mejora, el cambio y la superación cualitativa y cuantitativa de sus predecesoras, elevando los parámetros de calidad en imagen y sonido

- Digitalización de la imagen y sonido: lo que facilita su manipulación y distribución con parámetros más elevados de calidad y a costos menores de distribución, centrada más en los procesos que en los productos.

- Automatización e interconexión: pueden funcionar independientemente, su combinación permite ampliar sus posibilidades, así como su alcance.

- Diversidad: las tecnologías que giran en torno a algunas de las características anteriormente señaladas y por la diversidad de funciones que pueden desempeñar. 
Las innovaciones tecnológicas han permitido que se incluyan nuevos aspectos como la realidad aumentada, y la realidad virtual, que beneficia a la comunidad educativa (Urquiza, Auria, Daza, Carriel, \& Navarrete, 2016; Yánez, 2018), estas tecnologias son aplicadas desde un dispositivo móvil que se conecta para ejecutar el proceso totalmente ajenos al computador y que permitan al estudiante aprender y entretenerse a la vez.

Tomando en cuenta el aspecto del uso de equipos móviles para el aprendizaje surge el MLearning (Mobile Learning), que permite independencia del estudiante del uso de un equipo computador, (Chavarría \& García, 2012), y es que puede ser usado en diferentes areas de la vida cotidiana, dejando poco espacio al ocio. El M-Learning esta asociado a la mayoria de las teorias educativas desde la conductista hasta el aprendizaje colaborativo.

Los sistemas de gestion de aprendizaje (LMS) implementados con los e-learning permiten por medio de su adopción y el cumplimiento de estándares, que se transforman en unidades de innovación que permiten fomentar la educación superior con modalidades presencial, con soporte de aula virtual, semipresencial, virtual y a distancia (Pillo \& Bermudez, 2018, pág. 258), estos LMS son usados a nivel mundial por reconocidas universidades tanto en USA, Europa y América Latina la más predominante es MOODLE, siguiéndole Blackboard, teniendo una gran aceptación.

La inclusión de los LMS dentro de las universidades es considerada de mucho provecho para los actuantes del proceso enseñanza-aprendizaje ya que puede diversificar sus opciones para seguir en continua captación de conocimiento.

Finalmente se puede mencionar que esta investigación posee un objetivo general de considerar la gamificación como nueva herramienta pedagógica para el aprendizaje de las matemáticas. La metodología empleada se basó en un diseño bibliográfico con un tipo de investigación documental.

\section{Metodología}

Esta investigación consiste en un análisis teórico en base a los objetivos específicos, que son analizar cómo la gamificación permite mejorar el aprendizaje, y definir las características de la gamificación para el aprendizaje de las matemáticas. Por lo cual, la 
Marleni Mamani Sanchez

metodología empleada es de diseño bibliográfico de tipo documental. El diseño se fundamenta en la revisión sistemática, rigurosa y profunda de material documental de cualquier clase, donde se efectúa un proceso de abstracción científica, generalizando sobre la base de lo fundamental, partiendo de forma ordenada y con objetivos precisos (Palella Stracuzzi \& Martins Pestana, 2010).

La investigación documental se concreta exclusivamente en la recopilación de información de diversas fuentes, con el objeto de organizarla describirla e interpretarla de acuerdo con ciertos procedimientos que garanticen confiabilidad y objetividad en la presentación de los resultados (Palella Stracuzzi \& Martins Pestana, 2010). La composición de este artículo se creó a partir de una descripción general de la documentación especial sobre este tema, teniendo en cuenta las publicaciones de revistas indexadas y libros actuales relacionados.

\section{Resultados y discusión}

\section{Aprendizaje en las matemáticas}

Uno de las asignaturas que es difícil de comprender por la mayoría de los estudiantes es la matemática, pues la consideran con cierto nivel de dificultad, que les imposibilita poder resolver problemas matemáticos planteados en el aula de clases, debido a la concepción que se tiene de que esta asignatura es solo memorizar resultados y procedimientos (Ruiz, Alfaro, \& Gamboa, 2003, pág. 288), pero más allá de esta concepción la idea es entender que las matemáticas debe permitir desarrollar habilidades para el razonamiento matemático.

Según indica (Godino, Batanero, \& Font, 2003), cuando se toma en consideración el tipo de matemáticas que se quiere impartir y la forma como se desarrollará la enseñanza en los alumnos se debe reflexionar sobre dos puntos importantes de esta enseñanza que son:

- Que los alumnos lleguen a comprender y a apreciar el papel de las matemáticas en la sociedad, incluyendo sus diferentes campos de aplicación y el modo en que las matemáticas han contribuido a su desarrollo.

- Que los alumnos lleguen a comprender y a valorar el método matemático, esto es, la clase de preguntas que un uso inteligente de las matemáticas permite responder, 
las formas básicas de razonamiento y del trabajo matemático, así como su potencia y limitaciones (pág. 17)

Al igual que la lectura y la escritura, la matemática es fundamental en la educación elemental, por lo que es importante conocer las dificultades de aprendizaje existentes en los alumnos al momento de comprender matemáticas (Orrantia, 2006), y así conocer el nivel de éxito o fracaso que se tenga al momento de impartir la clase de manera que puedan tomarse previsiones y lograr que los alumnos adquieran conocimientos correctamente con el propósito de que sean capaces de enfrentar estos tiempo cambiantes sin temor.

Es por ello que las estrategias de enseñanza-aprendizaje son sumamente importantes y que el docente tome en consideración que la educación es integral viendo al individuo como un todo (mente, cuerpo y espíritu) (Herrera, Montenegro, \& Poveda, 2012), estimulando cada uno de estos aspectos en el alumno y lograr un aprendizaje efectivo para cada uno. Para ello es necesario tomar en cuenta aspectos como el ambiente familiar y cómo influye este en su capacidad de aprendizaje, el entorno sociocultural y socioeconómico, las relaciones interpersonales, puesto que el tema afectivo viene relacionado con el aprendizaje y como el estudiante es capaz de captar lo enseñado por el docente.

Las nuevas tecnologías también son utilizadas para la enseñanza de la matemática por lo que los docentes deben estar actualizados con el uso de cada una de estas herramientas tecnológicas para así poder aprovecharlas en el proceso, y es que como menciona (Herrera, et al., 2012) estas tecnologías hoy en dia son ineludibles pues al momento de que el estudiante desarrolle su vida profesional debera enfrentarse a diversas tecnologias en las empresas. No con esto se desea reemplazar al docente con la tecnología sino que estas serviran de apoyo para la enseñanza siempre y cuando estas sean utilizadas de manera adecuada.

\section{Gamificación en relación al aprendizaje y su aplicación en las matemáticas}

La gamificación de manera simple se puede definir como "una tecnología que permite el uso de mecánicas y técnicas de juego en entornos que son ajenos al juego" (Serrano Martinez, 2016), hoy en día ha tenido gran popularidad debido a que gracias a las nuevas 
tecnologías se han podido desarrollar herramientas que permiten al alumnado aprender de forma entretenida y diferente a la manera tradicional.

Según Jurado en el año 2017 y citado por (Zapata Vega, 2019) señala que:

Los beneficios de la gamificación se pueden plantear como oportunidades con relación al aprendizaje, ya que afianza la motivación intrínseca del actuante, puede elegir, controlar, colaborar, sentir el desafío y conseguir un resultado o logro. Se relaciona con el desarrollo cognitivo en la medida que se desarrollan habilidades para tomar decisiones, resolver problemas y para la autodeterminación (Zapata Vega, 2019, pág. 14)

A nivel pedagógico la gamificación es una técnica innovadora muy dinámica tanto para los alumnos como los docentes pues permite interactuar y adquirir conocimientos obteniendo diversas experiencias (Zapata Vega, 2019), pues esta especie de juegos serios otorgan potencial para desarrollar habilidades en los individuos que le servirán en su entorno laboral.

Algunas técnicas mecánicas de la gamificación según (Coello \& Gavilanes, 2019), son:

- Acumulación de los puntos: estos asignan un valor cuantitativo a diferentes acciones y se acumulan a medida que se realizan todo y cuando sean acertados.

- Escalando niveles: es un sistema donde se definen una serie de niveles de dificultad que se encuentra en una vía de escalada donde el usuario va superando para llegar al siguiente.

- Obtención de premios: a medida que se cumplen los objetivos se generan premios a modo de "colección".

- Regalos: son bienes que se le da al jugador de manera gratuita al conseguir un objetivo.

- Clasificaciones: se clasifica a los usuarios en función de los puntos u objetivos logrados.

- Desafíos: serían competiciones entre los usuarios, el mejor obtiene puntos o premios.

- Misiones o retos: conseguir resolver o superar un reto planteado, solo o en equipo. (págs. 16-17)

Así mismo algunas técnicas dinámicas expresadas por (Coello \& Gavilanes, 2019) que son más llamativas pues aumentan la motivación del usuario para seguir adelante logrando cumplir objetivos son:

- Recompensa: obtiene un beneficio.

- Estatus: se establece un nivel jerárquico.

- Logro: superación o satisfacción personal. 
- Competición: por el simple afán de competir e intentar ser mejor que el resto. (pág. 17)

De igual manera los componentes de la gamificación según (Hernández, 2019) son las recompensas por la ejecución de las dinámicas y mecánicas del juego y estos son:

- Avatares: representación visual del usuario.

- Colecciones: elementos para acumular.

- Tablas de clasificación: muestra visual de la progresión y logros de los jugadores.

- Niveles: pasos definidos para la progresión del usuario.

- Contenido bloqueado. (pág. 26)

La lúdica hace que cada ser humano tenga sus motivos para jugar y que sean el grado de competencia afianzado a un sujeto hábil y concreto para lograr desarrollar su pensamiento crítico frente a un objeto determinado, es decir, en el momento en el que alcanza el objetivo propuesto en la habilidad, se detiene un momento y reflexiona sobre lo antes pensado para tomar la decisión acertada (Coello \& Gavilanes, 2019, pág. 19)

Hay que tener presente que existe una diferencia entre la gamificación y los juegos educativos pues son estrategias completamente diferentes tanto en la teoría como en la práctica (Macías Espinales, 2017), la gamificación no significa jugar sino el empleo de elementos del juego (puntos, niveles, reglas, tiempo), pero no en contextos lúdicos con el fin de lograr ciertos objetivos o metas. El juego educativo se refiere a incorporar a los objetivos de aprendizaje el modo de juego de manera digital o no, y no necesariamente están relacionados al aprendizaje.

Como demostración de la adecuada conjugación de la tecnología y la gamificación (Macías Espinales, 2017) presenta cinco casos como apoyo para el aprendizaje en la Tabla 4.

\section{¿Por qué se gamifica?}

Como lo menciona Borras en 2015, citado por (Rojas Freire, 2019), existen ciertos puntos a favor en cuanto a la aplicación de la gamificación y se detalla a continuación en la Tabla

5.

\section{Tabla 4}

Conjugación de Tecnología y gamificación como apoyo al aprendizaje

\begin{tabular}{ccl}
\hline Caso & UNIVERSIDAD & \multicolumn{1}{c}{ DESCRIPCIÓN DEL CASO } \\
\hline \multirow{4}{1}{} & Universidad de & A través de un curso de segundo año que desarrolla habilidades para el diseño \\
Cape Town en & de juego en 2D, se empleó una narrativa que se basó en el subgénero \\
& Sudáfrica & Steampunk, en un pasado parecido a la era victoriana, pero con tecnología \\
\hline
\end{tabular}


Marleni Mamani Sanchez

\begin{tabular}{|c|c|c|}
\hline & & $\begin{array}{l}\text { avanzada. El curso fue dictado a través de una plataforma virtual que } \\
\text { conjugado con un ambiente gamificado (resolver misterios, conseguir pistas, } \\
\text { completar tareas, obtener puntos, entre otros) logró aumentar las } \\
\text { calificaciones de los estudiantes y un mayor compromiso }\end{array}$ \\
\hline 2 & $\begin{array}{l}\text { Universidad } \\
\text { Tecnológica de } \\
\text { Delft en Holanda }\end{array}$ & $\begin{array}{l}\text { A través de los cursos de computación y computación en la nube, constató } \\
\text { que trasladar el ambiente educativo tradicional a uno Gamificado } \\
\text { tecnológicamente, en el que se adecuó las actividades a las motivaciones de } \\
\text { los tipos de jugadores (exploradores, triunfadores, socializadores y } \\
\text { ganadores), no solo permitió mejorar el porcentaje de aprobados, también } \\
\text { evidenció mejoras en la interacción profesor alumno, en la evaluación positiva } \\
\text { de los estudiantes y en la participación voluntaria }\end{array}$ \\
\hline 3 & $\begin{array}{l}\text { Universidad de } \\
\text { Tecnología de } \\
\text { Lublin en } \\
\text { Polonia }\end{array}$ & $\begin{array}{l}\text { Empleó un sistema Gamificado con la ayuda de un software para dictar el } \\
\text { curso de Ingeniería de Software, en el tercer año de la carrera de Ciencias } \\
\text { Computacionales. Los estudiantes tenían el objetivo de completar tres } \\
\text { exámenes y un proyecto final para acreditar el curso, pero para lograrlo debían } \\
\text { realizar varias actividades; los estudiantes compitieron por sus calificaciones } \\
\text { y este sistema logró una clase con mayor asistencia, participación en } \\
\text { actividades adicionales y mejor tiempo de entrega de proyectos }\end{array}$ \\
\hline 4 & $\begin{array}{c}\text { Tecnológico de } \\
\text { Monterrey }\end{array}$ & $\begin{array}{l}\text { Los cursos de Liberalismo y expansión del primer año de preparatoria, fueron } \\
\text { adecuados a la plataforma Schoology con vocabulario propio de juego, por } \\
\text { ejemplo, los ejercicios se llamaron War Games y las tareas, Operations. Los } \\
\text { alumnos tenían la libertad de escoger la manera de realizar sus tareas ya sea } \\
\text { mediante podcasts, blogs, presentaciones o reportes. Se utilizó Kahoot y } \\
\text { Jeopardy para hacer preguntas de repaso que permitía que los estudiantes } \\
\text { ganaran puntos. El profesor empleó una hoja de cálculo de Google para } \\
\text { socializar la tabla de posiciones y los logros de cada participante. Al final del } \\
\text { curso, los estudiantes coincidieron que la Gamificación hizo la clase más } \\
\text { divertida y que los estimuló a desempeñarse mejor }\end{array}$ \\
\hline 5 & $\begin{array}{c}\text { Tecnológico de } \\
\text { Monterrey }\end{array}$ & $\begin{array}{l}\text { En el curso de inglés remedial I, se utilizó la narrativa storytelling y recursos } \\
\text { interactivos de portales como LearnEnglish de British Council, BBC News y } \\
\text { History Channel. Los alumnos trabajaron en equipo para culminar misiones, } \\
\text { que les permitían ganar puntos y medallas; se utilizó BookWidgets y quizzes } \\
\text { semanales para la revisión de contenido. Al finalizar el semestre los alumnos } \\
\text { incluyeron como actividades favoritas aquellas relacionadas con las misiones, } \\
\text { los juegos y el trabajo en equipo. Además, se logró una clase divertida que } \\
\text { facilitó la comprensión de los contenidos }\end{array}$ \\
\hline
\end{tabular}

Fuente: (Macías Espinales, 2017)

Tabla 5

Razones para aplicar la gamificación RAZONES

- Activa la motivación por el aprendizaje.

- La retroalimentación es constante.

- El Aprendizaje más significativo permitiendo mayor retención en la memoria al ser más atractivo.

- Genera compromiso con el aprendizaje y fidelización o vinculación del estudiante con el contenido y con las tareas en sí.

- Hay resultados más medibles (niveles, puntos y badges). 
- Genera competencias adecuadas y alfabetiza digitalmente.

- Los aprendices son más autónomos.

- Genera competitividad a la vez que colaboración.

- Genera conectividad entre usuarios en el espacio online

Fuente: (Rojas Freire, 2019)

Hoy resulta difícil para las instituciones y los docentes mantener interesados y enfocados a los estudiantes en el estudio debido a diversos factores de distracción que existen a su alrededor (equipos móviles, juegos de video entre otros), por ello es necesario diversificar la forma de aprendizaje sobre todo en aquellas asignaturas como la matemática.

Adicional a los aspectos antes mencionados se incluye el "estrés", que influye en muchas ocasiones en el aprendizaje en especial con la matemática pues se necesita pensar con claridad y tranquilidad con el propósito de comprender y razonar lógicamente lo que se trata de aprender (Salmerón Céspedes, 2017, pág. 8) de lo contrario el percibir sensaciones poco agradables durante la clase puede generar limitantes durante su estudio y poco avance en el mismo, a diferencia de aquellos alumnos que estén motivados y se sientan seguros de sus habilidades, adoptan estrategias de aprendizaje eficaces y obtienen buenos resultados en sus calificaciones.

No se puede obviar más la gran influencia que las variables afectivas ejercen en la construcción del conocimiento de los estudiantes. Hay que desentrañar los aspectos emocionales del aprendizaje, porque posiblemente en ellos se encuentre la raíz de muchos fracasos de la vida intelectual y, en particular, de la educación. (Salmerón Céspedes, 2017, pág. 8)

La gamificación juega un papel importante para mantener a los estudiantes motivados, y como lo expresa (Hernández, 2019) la motivación es un factor derivado de la gamificación donde se expresa que existen dos tipos de motivación detallados en la Tabla 6.

\section{Tabla 6}

Clasificación de la motivación en función de la gamificación

\begin{tabular}{cl}
\hline TIPO & \multicolumn{1}{c}{ CARACTERISTICAS } \\
\hline Motivación Intrínseca & $\begin{array}{l}\text { Es la que nace al interior del alumno, solo realiza la } \\
\text { actividad por placer de aprender y descubrir algo } \\
\text { nuevo. }\end{array}$ \\
\hline Motivación Extrínseca & $\begin{array}{l}\text { Es provocada por estímulos externos como premios, o } \\
\text { recompensas que ganará por realizar la actividad. }\end{array}$ \\
\hline
\end{tabular}

Fuente: (Hernández, 2019) 
Como ejemplo de la gamificación utilizada para el aprendizaje en las matemáticas se menciona el software Kahoot, que es gratuito y está orientado a la creación de cuestionarios para resolver preguntas online con la facilidad de utilizar cualquier dispositivo electrónico (Godoy Cedeño, 2020, pág. 15), se pueden crear cuestionarios de lógica matemática, para mejorar la aplicación de conocimientos, este se desarrolla en un ambiente virtual donde interactúan los participantes inculcando la competencia sana entre ellos.

El escape room es otro ejemplo de gamificación aplicado a la matemática donde interactúan de dos a seis amigos que entran en una habitación con alguna temática en específico, y el fin es poder escapar del lugar en un determinado tiempo estipulado (normalmente de 1 hora), (Salmerón Céspedes, 2017, pág. 15), deben explorar el entorno e ir progresando integrándose en el equipo con cada uno de los protagonistas, resolviendo enigmas, descifrando claves y encontrando pistas que les ayuda a desarrollar habilidades y lógica matemática.

Existe diversidad de juegos lúdicos que permiten a los estudiantes adquirir conocimientos y aumentar su aprendizaje por medio de este tipo de técnicas que facilitan su desarrollo cognitivo.

Como conclusión se puede indicar que la gamificación resulta interesante e importante para mejorar el aprendizaje en todos los niveles educativos pues ayuda en gran medida a la motivación de los estudiantes, así como la disminución del estrés presente en ellos producto del miedo a afrontar las clases que les parecen aburridas o difíciles y de diversos factores como socioculturales y económicos.

A pesar de que la gamificación posee desventajas en cuanto a la poca accesibilidad de la tecnología tanto para el estudiante como para los docentes y la poca habilidad tecnológica que tienen algunos, sus ventajas son mayores y generan aspectos positivos en el incremento del aprendizaje de los estudiantes.

En cuanto a la implementación de la gamificación en las matemáticas se ha observado una buena influencia de esta herramienta para desarrollar habilidades de cálculos y lógica matemática en los estudiantes pues les permite a través del uso de los diferentes software existentes desarrollar estrategias que les permitan avanzar de nivel, lograr cumplir las 
metas, con el propósito de alcanzar las mejores calificaciones los que los motiva a seguir siempre buscando mejorar sus capacidades para comprender cada tema de la materia.

\section{Referencias bibliográficas}

Aguado-Aguilar, L. (2001). Aprendizaje y memoria. . Revista de neurología, 32(4) , 373381.

Briceño, J. (2014). Reflexiones sobre la educación superior en Venezuela. Comunidady Salud, 12(1) , 69-73.

Castro, S., Guzmán, B., \& Casado, D. (2007). Las Tic en los procesos de enseñanza y aprendizaje. . Laurus, 13(23), 212-234.

Chavarría, M., \& García, I. (2012). M-Learning: Microblogging. Madrid-España. Universidad Autónoma de Madrid, 4-5. , 1-12.

Coello, L. J., \& Gavilanes, B. E. (2019). La gamificacion del proceso de enseñanza aprendizaje significativo. Guayaquil, Ecuador: Trabajo especial de grado de la Universidad de Guayaquil para optar al título de Licenciado en Ciencias de la Educación mención Sistemas Multimedia.

del Barrio, J. A., \& García, M. (2006). Las nuevas tecnologías como herramienta pedagógica para facilitar la comunicación. . Revista ICONO14 Revista científica de Comunicación y Tecnologías emergentes, 4(1) , 89-106.

Díaz, I. G. (2012). El juego lingüístico: una herramienta pedagógica en las clases de idiomas. . Revista de lingüística y lenguas aplicadas, (7) , 97-102.

Facundo, Á. H. (2004). La virtualización desde la perspectiva de la modernización de la educación superior: consideraciones pedagógicas. . International Journal of Educational Technology in Higher Education (ETHE), 1(1). , 1-17.

Godino, J. D., Batanero, C., \& Font, V. (2003). Fundamentos de la enseñanza y el aprendizaje de las matemáticas para maestros. Granada: Universidad de Granada.

Godoy Cedeño, C. E. (2020). Uso de la gamificación en el desarrollo del pensamiento lógico matemático en estudiantes de educación superior en una universidad privada de Lima, 2020. Lima, Perú: Trabajo especial de grado de la Universidad César Vallejo para optar al título de Doctor en Educación.

Hernández, A. (2019). La gamificación en aplicaciones móviles como apoyo para el aprendizaje de las matemáticas en la escuela secundaria. Mexicali, Baja California: Trabajo especial de grado de la Universidad Autónoma de Baja California para optar al título de Maestro en Educación. 
Herrera, N. L., Montenegro, W., \& Poveda, S. (2012). Revisión teórica sobre la enseñanza y aprendizaje de las matemáticas. Revista virtual universidad católica del norte, (35) , 254-287.

Iglesia, J. (2008). Los cuentos motores como herramienta pedagógica para la educación infantil y primaria. . ICONO 14, Revista de comunicación y tecnologías emergentes, $6(1), 1-15$.

Macías Espinales, A. V. (2017). La Gamificación como estrategia para el desarrollo de la competencia matemática: plantear y resolver problemas. Guayaquil, Ecuador: Trabajo especial de grado de la Universidad Casa Grande para optar al título de Magister en Tecnología e Innovación Educativa.

Martínez, R., Arrieta, X., \& Meleán, R. (2012). Desarrollo cognitivo conceptual y características de aprendizaje de estudiantes universitarios. . Omnia, 18(3) , 35-48.

Muñiz, L., Alonso, P., \& Rodríguez, L. J. (2014). El uso de los juegos como recurso didáctico para la enseñanza y el aprendizaje de las Matemáticas: estudio de una experiencia innovadora. Unión. Revista Iberoamericana de Educación Matemática, $39,19-33$.

Orrantia, J. (2006). Dificultades en el aprendizaje de las matemáticas: una perspectiva evolutiva. Revista da Associação Brasileira de Psicopedagogia 23(71) , 1-34.

Palella Stracuzzi, S., \& Martins Pestana, F. (2010). Metodología de la investigación cuantitativa. Caracas, Venezuela: FEDUPEL, Fondo Editorial de la Universidad Pedagógica Experimental Libertador.

Pillo, D. M., \& Bermudez, L. A. (2018). Estudio de innovación tecnológica aplicada en procesos formativos con sistemas de gestión de aprendizaje (LMS) en la educación universitaria del Ecuador. Revista Ibérica de Sistemas e Tecnologias de Informação, (E15) , 256-270.

Rodríguez, R. M. (2011). Repensar la relación entre las TIC y la enseñanza universitaria: problemas y soluciones. Profesorado 15(1) , 9-22.

Rojas Freire, C. E. (2019). Estrategias de gamificación para el desarrollo de la Inteligencia lógico-matemática de los estudiantes de sexto año de eduación general básica de la Unidad Educativa Atahualpa. Ambato, Ecuador: Trabajo especial de grado de la Universidad Tecnológica de Indoamerica para optar al título de Maestría en Educación mención Innovación y Liderazgo.

Ruiz, Á., Alfaro, C., \& Gamboa, R. (2003). Aprendizaje de las matemáticas: conceptos, procedimientos, lecciones y resolución de problemas. . Uniciencia, 20 , 285-296.

Salmerón Céspedes, I. (2017). Escape Classroom. Propuesta didáctica motivadora para la clase de Matemáticas. Almería: Trabajo especial de grado de la Universidad de Almería para optar al título de master en Profesorado de Educación Secundaria Obligatoria, Bachillerato, Formación Profesional y Enseñanza de Idiomas. . 
Marleni Mamani Sanchez

Serrano Martinez, C. J. (2016). Gamificacion Universitaria. Jaén: Trabajo especial de grado de la Universidad de Jaén para obtener el título de Ingeniería en Informática.

Urquiza, L. I., Auria, B., Daza, S., Carriel, F. D., \& Navarrete, R. (2016). Uso de la realidad virtual, en la educación del futuro en centros educativos del Ecuador. . Journal of Science and Research: Revista Ciencia e Investigación, 1(4) , 26- 30.

Valle, A., González, R., Cuevas, L. M., \& Fernández, A. P. (1998). Las estrategias de aprendizaje: características básicas y su relevancia en el contexto escolar. Revista de psicodidáctica, (6) , 53-68.

Yánez, Á. S. (2018). La realidad aumentada y su relación en los procesos pedagógicos de los estudiantes universitarios. Ambato, Ecuador: Trabajo especial de grado de la Universidad Técnica de Ambato para optar al título de Licenciado en Ciencias de laEducación, Mencion Informática y Computación.

Zapata Vega, Z. M. (2019). Estrategias metodológicas de la gamificacion en el aprendizaje . Guayaquil, Ecuador: Trabajo especial de grado de la Universidad de Guayaquil para optar por el título de Licenciada en Educación Primaria. 